\title{
Análise espacial, clínico-epidemiológica e laboratorial de crianças internadas com leishmaniose visceral no Pará/Amazônia Brasileira
}

Spatial, clinical-epidemiological and laboratory analysis of children admitted with visceral leishmaniasis in Pará/ Brazilian Amazon

Análisis espacial, clínico-epidemiológico y de laboratorio de niños admitidos con leishmaniasis visceral en Pará/ Amazonia Brasileña

\section{RESUMO}

A Leishmaniose Visceral (LV), é uma infecção sistêmica que afeta pessoas no mundo todo. É endêmica no Pará. Objetivo: Analisar as características espaciais, clínico-epidemiológicas, laboratoriais, tratamento e letalidade de crianças com LV em hospital de referência do Pará. Método: Estudo retrospectivo-analítico, transversal, com abordagem quantitativa dos prontuários de crianças com LV internados entre 2012 e 2016 . Resultado: Os casos predominaram na zona rural $(77,1 \%$ e infecção primária (86,5\%). Os menores de 6 anos e do sexo masculino foram os mais acometidos. A distribuição espacial dos casos foi heterogênea, predominando o Nordeste Paraense (80,2\%). A confirmação laboratorial foi a mais utilizada (86,5\%). N-metil glucamina foi a terapêutica de eleição (89,3\% dos casos). Conclusão: Houve cura em mais de $90 \%$ dos casos. A letalidade associou-se à sangramentos, à plaquetopenia, à falha no tratamento inicial, ao tempo de tratamento e ao maior escore sistema de avaliação de risco de vida (SARV).
\end{abstract}

DESCRITORES: Leishmaniose Visceral; Crianças; Epidemiologia; Letalidade.

\begin{abstract}
Visceral Leishmaniasis (VL) is a systemic infection that affects people worldwide. It is endemic in Pará. Objective: To analyze the spatial, clinical-epidemiological, laboratory, treatment and lethality characteristics of children with VL in a reference hospital in Pará. Method: Retrospective-analytical, cross-sectional study, with a quantitative approach to medical records of children with VL hospitalized between 2012 and 2016. Result: Cases predominated in rural areas (77.1\%) and primary infection (86.5\%). Children under 6 years old and males were the most affected. The spatial distribution of cases was heterogeneous, with a predominance of Northeast Pará (80.2\%). Laboratory confirmation was the most used (86.5\%). N-methyl glucamine was the therapy of choice (89.3\% of cases). Conclusion: There was a cure in more than $90 \%$ of cases. Mortality was associated with bleeding, thrombocytopenia, initial treatment failure, treatment time and higher life risk assessment system (SARV) score.
\end{abstract}

DESCRIPTORS: Visceral Leishmaniasis; Children; Epidemiology; Lethality.

\section{RESUMEN}

La leishmaniasis visceral (LV) es una infección sistémica que afecta a personas en todo el mundo. Es endémica en Pará.Objetivo: Analizar las características espaciales, clínico-epidemiológicas, de laboratorio, de tratamiento y letalidad de niños con LV en un hospital de referencia en Pará.Método: Estudio retrospectivo-analítico, transversal, con enfoque cuantitativo historias clínicas de niños con LV hospitalizados entre 2012 y 2016. Resultado: Predominaron los casos en el área rural (77,1\%) y la infección primaria (86,5\%). Los niños menores de 6 años y los varones fueron los más afectados. La distribución espacial de los casos fue heterogénea, con predominio del Noreste de Pará (80,2\%). La confirmación de laboratorio fue la más utilizada (86,5\%). La N-metil glucamina fue la terapia de elección (89,3\% de los casos). Conclusión: hubo cura en más del 90\% de los casos. La mortalidad se asoció con hemorragia, trombocitopenia, fracaso del tratamiento inicial, tiempo de tratamiento y mayor puntuación del sistema de evaluación de riesgos de vida (SARV).

DESCRIPTORES: Leishmaniasis Visceral; Niños; Epidemiología; Letalidad.

RECEBIDO EM: 06/16/2021 APROVADO EM: 06/25/2021

\section{Jorge Luís Monteiro Spinelli}

Mestrado Epidemiologia e Vigilância em Saúde pelo Instituto Evandro Chagas (IEC/PA). Instituto Evandro Chagas, Brasil. ORCID: 0000-0001-8466-6819 


\section{Ana Maria Revorêdo da Silva Ventura}

Doutorado em Medicina Tropical pela Fundação Oswaldo Cruz (FIOCRUZ). Instituto Evandro Chagas, Brasil. ORCID: 0000-0002-4737-1533

\section{Fernando Tobias Silveira}

Pós-doutorado pela Faculdade de Medicina da Universidade de São Paulo (FMUSP). Instituto Evandro Chagas, Brasil. ORCID: 0000-0002-0412-6060

\section{Breno Caldas Ribeiro}

Graduação em Fisioterapia pela Universidade do Estado do Pará (UEPA). Universidade do Estado do Pará, Brasil. ORCID: 0000-0002-6355-8605

\section{Sara Elly Dias Nunes}

Graduada em Fisioterapia pela Universidade da Amazônia (UNAMA). Universidade da Amazônia, Brasil. ORCID: 0000-0002-3704-7246

\section{Rebecca Costa da Silva}

Graduada em Fisioterapia pela Universidade da Amazônia (UNAMA). Universidade da Amazônia, Brasil. ORCID: 0000-0002-4483-3202

\section{INTRODUÇÃO}

A Leishmaniose Visceral (LV), também conhecida como Calazar ou Leishmaniose Americana, é uma infecção sistêmica grave causada por um protozoário do gênero leishmania que afeta dois milhões de pessoas por ano. Atualmente, a Organização Mundial de Saúde (OMS) considera a LV uma das sete endemias mundiais de absoluta prioridade dentre as Doenças Tropicais Negligenciadas (DTN's). É um grande problema de saúde pública, devido ao seu caráter endêmico em várias regiões do mundo e seu alto potencial de letalidade. Dentre os 7 países mais afetados estão Bangladesh, Brasil, Índia, Etiópia, Quênia, Nepal e Sudão, que juntos representam aproximadamente $90 \%$ dos casos ${ }^{1}$.

No mundo, estima-se sua incidência em 500.000 casos novos e 50.000 mortes a cada ano. Nas Américas, o Brasil é o país de maior endemia, abrangendo aproximadamente 97\% de todos os casos no continente ${ }^{2}$.

No Brasil, o principal vetor da LV é o Lutzomya longipalpis conhecido como mosquito-palha ou birigui que transmite a espécie Leishmaniose chagasi aos seres vertebrados. O cão doméstico é o principal reservatório da leishmania em áreas urbanas, o que constitui um fator agravante ao se levar em consideração a relação direta dos seres humanos (hospedeiro final) com os cães ${ }^{3}$.

No Brasil, a importância da LV consiste na sua elevada Incidência (3.829 casos, em 2015) e sua ampla distribuição com registro da doença no Nordeste (1.806 casos); Sudeste (538 casos); Norte (469 casos); Centro-Oeste (157 casos); e Sul (5 casos) ${ }^{4}$.

No Brasil, a LV teve sua origem na área rural, mas devido à falta de saneamento básico e condições precárias de moradia vinculadas ao processo desordenado de urbanização do país, a doença avançou para os subúrbios das grandes cidades brasileiras, nos últimos 40 anos, como atestam relatos clínicos ou inquéritos sorológicos, semelhante ao que ocorre em outros paí$\operatorname{ses}^{5,6}$.

Por sua ocorrência na zona rural e na periferia das grandes cidades, a LV costuma atingir indivíduos de baixo poder aquisitivo. Essa particularidade social pode interferir no estado de saúde da população pelas condições sanitárias precárias que vivem, em que as infecções e a desnutrição agravadas pela doença são fatores de mau prognóstico ${ }^{6}$.

Em 2014, no Brasil, dos cerca de 1.980 casos de LV notificados por ano, um pouco mais da metade $(54,4 \%)$ acometeu crianças menores de 10 anos e, desses, $41 \%$ pertenciam a faixa etária de menores de cinco anos. As crianças além de serem mais susceptíveis estão mais expostas ao vetor no peridomicílio, além de serem mais pro- pensas à desnutrição, condição que piora o quadro clínico da leishmaniose ou é por ela agravado?

O Pará é um dos estados do país que contribui de modo significativo para a expansão da doença, com 1.266 casos notificados entre os anos de 2010 a $2013^{8}$.

Dentre os fatores que contribuem para a expansão da LV no país, citam-se a deficiência ou ausência de serviços de saúde nas localidades dos casos índices, capacitação inadequada dos profissionais de saúde quanto suspeição diagnóstica e controle ineficaz do gerenciamento de recursos para diagnóstico e tratamento. Esse panorama predispõe o encaminhamento dos indivíduos doentes para tratamento hospitalar ${ }^{8}$.

Nesse contexto, o objetivo deste trabalho é analisar as características espaciais, clínico-epidemiológicas, laboratoriais, tratamento e letalidade de crianças com LV em hospital de referência do Estado do Pará.

\section{MÉTODOS}

Trata-se de um estudo documental, retrospectivo-analítico, transversal, com abordagem quantitativa realizado no Hospital Universitário João de Barros Barreto (HUJBB), uma unidade de assistência, ensino e pesquisa, integrada ao Complexo Hospitalar da Universidade Federal do Pará (UFPA), referência no Pará, locali- 
zado na capital do estado, Belém, região amazônica brasileira, o qual se caracteriza por oferecer à população serviço público (Sistema Único de Saúde - SUS) de pronto atendimento, apoio diagnóstico, clínica médica, pediatria, infectologia, serviço de referência aos portadores de HIV e de tratamento para tuberculose e leishmaniose.

A coleta de dados foi realizada no setor de Divisão de Arquivo Médico e Estatístico (DAME) nos meses de maio a julho de 2019. Os dados foram obtidos mediante análise dos prontuários, seguido por preenchimento de uma ficha de avaliação individual com identificação, procedência, tempo de doença, dados clínicos e laboratoriais, patologias associadas, uso de medicamento forma de diagnóstico, exames laboratoriais, tipo e período de tratamento e desfecho clínico. Também foram identificadas as crianças com LV com risco de evolução para situações de gravidade e para o óbito, segundo a recomendação oficial do SARV, em ficha adaptada do Guia de Vigilância em Saúde (BRASIL, 2017a).

Os critérios de inclusão foram crianças e adolescentes na faixa etária de 0 a 12 anos internados no HUJBB com diagnóstico confirmado, clínico e/ou laboratorialmente de LV no período de 01 de janeiro de 2012 a 31 de dezembro de 2016. Foram excluídos os prontuários de pacientes com LV que apresentavam informações insuficientes para preenchimento do protocolo elaborado pelo pesquisador ou informações inelegíveis ou insuficientes para preenchimento.

$\mathrm{Na}$ construção da base cartográfica houve colaboração do LABGEO (Laboratório de Geoestatística) da Universidade do Estado do Pará (UEPA) para geração dos mapas temáticos afim de expressar as relações espaciais e temporais dos casos de $L V$, além de utilização de fontes secundárias de domínio público para criação de mapas cartográficos: a projeção SIRGAS 2000 e o banco de dados do Censo 2010 (dados populacionais), ambas disponibilizadas pelo Instituto Brasileiro de Geografia e Estatística (IBGE). A classificação das mesorregiões do Pará seguiu as definições do IBGE.
Os dados foram

obtidos mediante

análise dos

prontuários, seguido

por preenchimento

de uma ficha de

avaliação individual

com identificação,

procedência,

tempo de doença,

dados clínicos

e laboratoriais,

patologias

associadas, uso de

medicamento forma

de diagnóstico,

exames laboratoriais,

tipo e período

de tratamento e

desfecho clínico.
Realizou-se a depuração dos dados pelo Programa Tabwin (DATASUS/MS, disponível em: http://datasus.saude.gov. br para a retirada de possíveis fatores causadores de vieses, como incompletudes, redundâncias e inconsistências dos dados analisados. Seguindo-se o processo de depuração ocorreu a implementação das coordenadas geográficas, as quais foram indexadas ao banco de dados dos casos notificados na área e período de estudo.

Elaborou-se uma planilha eletrônica, para armazenamento dos dados, no software Microsoft Excel ${ }^{\circ}$ 2010. Para representação descritiva dos dados, utilizaram-se tabelas (Software Microsoft Word ${ }^{\circ}$ ) em que as variáveis numéricas foram representadas por médias, desvio-padrão ou por mediana, $1^{\circ}$ quartil, $3^{\circ}$ quartil e valor mínimo e máximo dessas variáveis. As variáveis categóricas foram descritas em frequências absolutas e relativas.

A análise dos dados foi processada utilizando-se o programa Epi Info 7.2 e o programa Jamovi versão 0.9.5.15 para comparação das médias nos grupos óbito e sobreviventes. Utilizou-se o Teste t-Student (variáveis com distribuição normal) ou o teste de Mann-Whitney (variáveis com distribuição não paramétrica). Para identificar a dependência entre as variáveis clínicas (independente) e o óbito (dependente) por leishmaniose utilizou-se o Teste Exato de Fisher. Adotou-se nível de significância de $5 \%$ (p valor $\leq 0,05$ ) em todos os testes estatísticos.

Técnicas de geoprocessamento em ambientes ArcGis 10.5 e TerraView 4.0 foram utilizadas para análise da distribuição espacial dos casos de LV identificados no HUJBB, segundo o local de infecção: técnica de Fluxo (para acesso dos pacientes até o serviço), técnica geoestatística de Kernel (identificação dos locais com maiores concentrações de casos) e álgebra de mapas (expressão visual do inter-relacionamento das bases georreferenciadas dos dados clínicos, epidemiológicos, e de políticas públicas).

Este estudo foi aprovado no Comitê de Ética em Pesquisa (CEP) do Instituto Evandro Chagas (IEC) em 21 de março 
Tabela 1 - Dados sócios-demográficos das crianças com LV Internadas no HUJBB no período de 2012 a 2016, Belém, Pará

\section{VARIÁVEIS}

ZONA

Urbana

Rural

Total

TIPO DE ENTRADA

Caso novo

77

12

Recidiva

Total

SEXO

Masculino

59

37

Feminino

Total

AUTÓCTONE

Sim

Não

Total

IDADE

0 a 6 anos

7 a 9 anos

$\geq 10$ anos

Total

Fonte: Dados da pesquisa

N

22

96

22

89

96

60

36

96
74

$86,5 \%$

$13,5 \%$

$100 \%$

$62,5 \%$

$37,5 \%$

$100 \%$

Figura 1 - Distribuição Espacial de casos de LV no estado do Pará, atendidos pelo Hospital de referência, no periodo de janeiro de 2012 a dezembro de 2016. Belém, Pará.

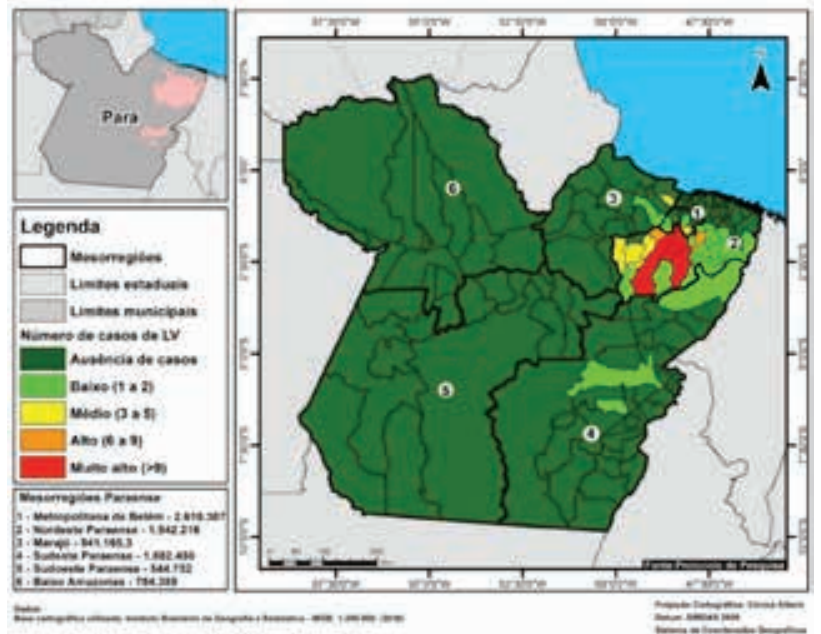

de 2019 (parecer no 3.213.985, CAAE 08390919.5.0000.0019).

\section{RESULTADOS}

Identificaram-se 127 casos com LV no período do estudo, com análise de 109 prontuários (18 prontuários foram danificados e/ou perdidos devido problemas estruturais no prédio, após fortes chuvas). Do total disponibilizado (109), 13 preencheram os critérios de exclusão, permanecendo então 96 prontuários elegíveis.

O tempo médio de doença foi de 71,5 dias. A Tabela 1 mostra os dados sócios demográficos da pesquisa, com maioria dos casos provenientes da zona rural $(77,1 \%)$, a predominância de infecções primárias (86,5\%), 61,5\% eram do sexo masculino; $62,5 \%$ eram autóctones. O maior acometimento $(83,3 \%)$ ocorreu em menores de 6 anos, seguido de $14,6 \%$ entre 7 a 9 anos e de 2,1\% em maiores de 10 anos.

A distribuição espacial foi heterogênea nas mesorregiões do estado do Pará (Figura 1): o Nordeste Paraense apresentou maior número de ocorrência, com 80,2\% oriundos de diversos municípios, distribuídos principalmente em Acará, Moju e Tome-Açu (10,4\%), com 10 casos cada, São Domingos do Capim (9,4\%), com 9 casos e Abaetetuba (7,3\%) com 7 casos.

As principais manifestações clínicas apresentadas foram: febre $(97,8 \%)$, astenia $(78,8 \%)$, palidez $(90,6 \%)$, esplenomegalia $(95,5 \%)$, hepatomegalia $(94,4 \%)$. O critério laboratorial foi o mais utilizado para confirmação da doença $(86,5 \%)$, com destaque para a imunofluorescência indireta (IFI) $(91,1 \%)$. O tratamento com N-dimetil glucamina foi realizado em $89,3 \%$ das crianças, com evolução para cura em sua quase totalidade (92,7\%), (Gráfico 1).

A Tabela 2 mostra que o sangramento [não especificado o(s) local(is) de sangramento na maior parte dos prontuários] foi a única das manifestações clínicas que teve influência significativa sobre o óbito ( $\mathrm{p}=0,05 ; \mathrm{OR}=10,1$; IC 95\%: 1,3 - 79,4).

$\mathrm{Na}$ tabela 3 encontram-se os parâmetros laboratoriais entre as crianças e adolescentes que evoluíram para cura e 
Gráfico 1 - Evolução dos casos das crianças com LV Internadas no HUJBB no período de 2012 a 2016, Belém, Pará.

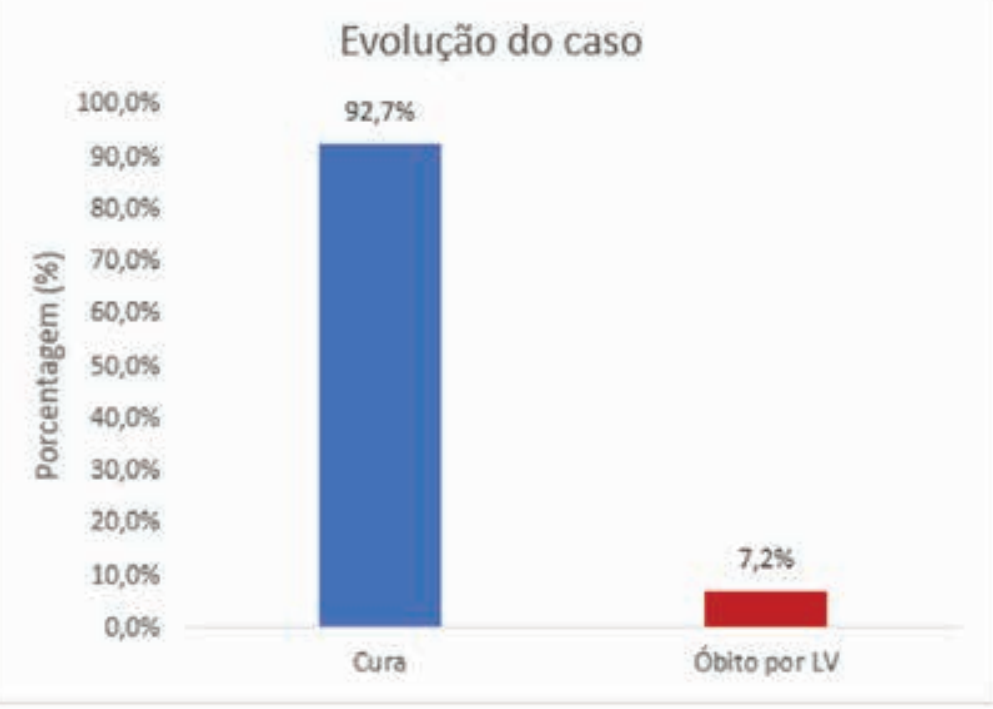

Fonte: Dados da pesquisa.

Tabela 2 - Manifestações clínicas vs óbito das crianças e adolescentes com LV Internadas no HUJBB no período de 2012 a 2016, Belém, Pará.

$\begin{array}{lccc}\text { VARIÁVEIS } & \text { ÓBITO (\%) } & \text { OR (IC 95\%) } & \text { P-VALOR } \\ \text { FEBRE } & & & \\ \text { Sim } & 7,9 \% & - & 0,99^{*} \\ \text { Não } & 0 \% & & \end{array}$

FRAQUEZA

$\operatorname{Sim}$

$7,4 \%$

Não

$5,5 \%$

$1,3(0,14-12,5)$

$0,99 *$

PALIDEZ

$\operatorname{Sim}$

Não

$7,8 \%$

$12,5 \%$

$0,6(0,06-5,6)$

$0,51^{*}$

EDEMA

Sim

$15,6 \%$

Não

$2,4 \%$

$7,5(0,84-68,6)$

$0,07^{*}$

EMAGRECIMENTO

Sim

$6 \%$

Não

$9,9 \%$

$0,6(0,1-3,1)$

$0,67^{*}$

ICTERÍ́CIA

Sim

Não

$5,2 \%$

$7,1 \%$

$0,7(0,07-6,8)$

$0,99 *$

ESPLENOMEGALIA

Sim

$8,2 \%$

$0,99 *$ àquelas que foram à óbito, em que se observa que apenas o hematócrito $(\mathrm{p}=0,05)$ e a contagem de plaquetas $(p=0,03)$ foram significativamente menores no grupo que evoluiu para óbito.

As crianças que receberam tratamento para LV com anfotericina B tiveram significativa letalidade se comparada com àquelas que receberam $\mathrm{N}$-metil glucamina como primeira opção terapêutica $(\mathrm{p}<$ 0,00; $\mathrm{OR}=40,5$; IC 95\%: 62-263), fato não observado dentre os que apresentaram ou não falência ao tratamento (Tabela 4).

O escore médio do sistema de avaliação de risco de vida (SARV) foi de 1,6 $\pm 1,5$, próximo do escore 2 de maior risco conforme determinado pelo Guia de Vigilância em Saúde?.

Nesta casuística do HUJBB, as crianças e adolescentes que evoluíram à óbito tiveram uma média significativamente maior do escore SARV do que as que evoluíram para a cura (3,5 vs 1,$4 ; \mathrm{p}=0,00)$.

\section{DISCUSSÃO}

A Leishmaniose é uma Doença Tropical Negligenciada (DNT) que constitui um problema de saúde pública mundial, considerada pela OMS como uma das cinco doenças infecto-parasitárias endêmicas de maior relevância, presente em aproximadamente 98 países ${ }^{9,10}$. O Brasil apresenta $95 \%$ dos casos registrados de LV na América Latina, em franco crescimento, tanto em magnitude como em expansão geográfica, com registro em todos os Estados, sendo o Pará um dos que possuem maior incidência da doença ${ }^{11,12}$. Neste sentido, os resultados obtidos neste estudo oferecem subsídios importantes para elaboração de medidas estratégicas de combate à doença.

No presente estudo houve um predomínio de internações por LV advindas da zona rural $(75,2 \%)$, contraponto ao estudo de Silva et al. (2017) ${ }^{13}$ que identificaram na cidade de Palmas, Tocantins, uma maior concentração de casos oriundos da zona urbana com $98,2 \%$.

Para a maioria $(86,5 \%)$ das crianças e adolescentes com LV tratava-se de infec- 


$\begin{array}{lccc}\text { Não } & 0 \% & - & 0,99^{*} \\ \text { HEPATOMEGALIA } & & & \\ \text { Sim } & 8,3 \% & 0,99^{*} \\ \text { Não } & 0 \% & \\ \text { SANGRAMENTO } & & & \\ \text { Sim } & 33,3 \% & 10,1(1,3-79,4) & 0,05^{*} \\ \text { Não } & 4,7 \% & \\ \text { OR = Odds Ratio, IC } 95 \% \text { = Intervalo de confiança (95\%), }{ }^{*} \text { Teste Exato de Fisher. Fonte: Dados da Pesquisa. } & \end{array}$

ção primária (casos novos), percentual inferior aos $91,8 \%$ de casos novos observados por Silva e Gaioso $(2013)^{14}$, ao realizarem pesquisa de LV no Pará, no período de 2007 a 2011. Os casos de recidiva desse estudo foram de $13,5 \%$, percentual superior aos 2,5\% (43/1.738 casos) observado no estudo dos autores supracitados.

Todos os casos de LV atendidos no HUJBB ocorreram na faixa etária pediá- trica, o que caracteriza a vulnerabilidade deste grupo à afecção. No estudo sobre LV no Estado do Pará, entre os anos de 2007 a 2011, as crianças constituíram a população particularmente susceptível, com progressão do estado mórbido em decorrência da imaturidade do sistema imunológico ${ }^{14}$.

Os casos de LV em 83,3\% foram em menores de 6 anos e 14,6\% entre 7 a 9 anos, similar ao observado por Muller et al. $(2008)^{15}$ e inferior a cerca de $60 \%$ em crianças menores de quatro anos em um levantamento do número de casos de LV no Brasil, nos anos de 2009 a $2013^{16}$.

O predomínio no sexo masculino $(61,5 \%)$ corrobora outros estudos ${ }^{17,18}$. Segundo os autores citados, fatores hormonais e a tendência desse gênero em realizar atividades fora do domicílio contribuiria para maior chance de exposição aos flebotomíneos vetores da doença.

Os casos autóctones desta pesquisa representaram 62,5\%, inferior aos $86 \%$ evidenciado por Silva e Gaioso (2013) ${ }^{14}$, em estudo realizado no Pará com informações referentes aos dados epidemiológicos dos casos de LV notificados no estado entre janeiro de 2007 a dezembro de 2011, que também acometeu mais a população pediátrica.

Tabela 3 - Letalidade relacionada aos dados laboratoriais das crianças e adolescentes com LV internadas no HUJBB no período de 2012 a 2016, Belém, Pará.

\begin{tabular}{|c|c|c|c|c|c|}
\hline \multirow{2}{*}{ VARIÁVEL } & \multicolumn{2}{|c|}{ NÃO ÓBITO } & \multicolumn{2}{|c|}{ ÓBITO } & \multirow{2}{*}{ P-VALOR } \\
\hline & MÉDIA ×DP * & MEDIANA (Q3 - Q1)** & MÉDIA ×DP* & MEDIANA (Q3 - Q1)** & \\
\hline $\mathrm{Hb}$ & $8,1 \pm 1,6$ & $7,9(9,1-6,8)$ & $6,9( \pm 2)$ & $7,5(8,7-5,8)$ & $0,09^{* * * *}$ \\
\hline Leucócitos & $4161,3 \pm 2721,8$ & $3420(5000-2430)$ & $3021,5( \pm 1713,9)$ & $2820(3880-1720)$ & $0,31^{* * *}$ \\
\hline Plaquetas (mil/mm3) & $124,6 \pm 122,9$ & $80,3(125-50)$ & $53,9( \pm 54,9)$ & $27(98,4-17,3)$ & $0,03^{* * *}$ \\
\hline
\end{tabular}

Tabela 4 - Tipo, falência e duração de tratamento vs óbito em crianças e adolescentes com LV internadas no HUJBB no período de 2012 a 2016, Belém, Pará.

\begin{tabular}{|c|c|c|c|c|c|}
\hline VARIÁVEIS & \multicolumn{2}{|r|}{ ÓBITO (\%) } & OR (IC 95\%) & \multicolumn{2}{|c|}{ P-VALOR } \\
\hline \multicolumn{6}{|l|}{ TRATAMENTO INICIAL } \\
\hline N-metil glucamina & \multicolumn{2}{|r|}{$2,4 \%$} & $40,5(62-263)$ & \multicolumn{2}{|c|}{$0,00^{*}$} \\
\hline Anfotericina B & \multicolumn{2}{|r|}{$50 \%$} & & & \\
\hline \multicolumn{6}{|l|}{ FALENCIA NO TRATAMENTO INICIAL } \\
\hline Sim & \multicolumn{2}{|r|}{$4,7 \%$} & $0,6(0,06-6,3)$ & \multicolumn{2}{|c|}{$0,99^{*}$} \\
\hline Não & \multicolumn{2}{|r|}{$7 \%$} & & & \\
\hline \multicolumn{6}{|c|}{ OR = Odds Ratio, IC 95\% = Intervalo de confiança (95\%), ${ }^{*}$ Teste Exato de Fisher. Fonte: Dados da Pesquisa } \\
\hline \multicolumn{6}{|c|}{$\begin{array}{l}\text { Tabela } 5 \text { - Sistema de avaliação de risco (SARV) vs Letalidade em crianças e adolescentes com LV internadas no } \\
\text { HUJBB no período de } 2012 \text { a 2016, Belém, Pará. }\end{array}$} \\
\hline \multirow[t]{2}{*}{ VARIÁVEL } & $\mathbf{N}$ & NÃO ÓBITO & \multicolumn{2}{|r|}{ ÓBITO } & P-VALOR \\
\hline & & 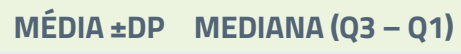 & MÉDIA \pm DP & MEDIANA (Q3 - Q1) & \\
\hline Sistema de avaliação de risco de vida & 96 & $1(2-0)$ & $3,5 \pm 1,7$ & $4(5-2)$ & $0,00^{* *}$ \\
\hline
\end{tabular}


A maioria dos casos de LV internados no HUJBB no período deste estudo eram oriundos da Mesorregião do Nordeste do Pará, com destaque para os municípios do Acará, Moju e Tomé-açu, diferentemente da capital paraense que teve poucos casos. Condições sanitárias inadequadas referente ao fornecimento e tratamento de água e do lixo são uma realidade nesses municípios, o que atraem cães vadios que são o principal reservatório da doença ${ }^{19,20}$. Outro agravante é a dificuldade de acesso aos serviços médicos e hospitalares por limitações ou deficiência no sistema de transporte público de uma grande parcela da população dos que vivem nessas áreas, o que contribuiria para agravar a doença com consequente hospitalização ${ }^{21}$.

Os dados georreferenciados a partir dos casos de LV em crianças e internadas no HUJBB demonstram a presença da doença no Estado do Pará e a necessidade de seu controle, o que inclui transpor as dificuldades climáticas e físico-geográficas da própria Amazônia, melhorar a gestão e o orçamento dos recursos destinados para esse fim, e, maior investimento na qualificação de profissionais para a análise e divulgação dos dados relacionados à Leishmaniose. Desse modo, haverá mais eficiência e agilidade na demarcação de áreas de risco, com repasse dessas informações às autoridades competentes a aos profissionais de saúde ${ }^{18,22}$.

A febre $(97,8 \%)$ foi a principal manifestação clínica nos pacientes com LV internadas no HUJBB. Kumar et al. $(2018)^{23} \mathrm{e}$ Cunha et al. (2019) $)^{24}$ também destacaram como os sinais e sintomas mais comuns a febre prolongada, astenia, perda de peso e hepatoesplenomegalia. $\mathrm{O}$ aumento do fígado e do baço (respectivamente, 94,4\% e $95,5 \%$ ) se seguiram em maior frequência depois da febre. A esplenomegalia é um dado relevante para suspeição clínica da doença ${ }^{25}$, que costuma estar ausente nos imunodeprimidos ${ }^{26}$.

O diagnóstico laboratorial (sorológico e parasitológico) é suficiente para confirmação de LV27, tendo predominado nesse estudo $(86,5 \%)$ semelhante $(88,8 \%)$ a outra pesquisa realizada no Pará entre 2007 a 2011, cujos casos também tiveram diagnóstico laboratorial ${ }^{14}$.

Dentre os exames laboratoriais, a principal forma de diagnóstico da LV foi por IFI. Atualmente, este é o teste disponibilizado pelo $\mathrm{SUS}^{28}$ e permanece como um dos mais utilizados nos principais laboratórios públicos de referência do Brasil, apesar do advento de novos métodos mais sensíveis para identificação da doença ${ }^{29}$. O IFI evidencia a presença de anticorpos IgG anti-leishmania, todavia, não assegura a presença ativa do parasito e assim, na presença de um resultado reagente é necessário associar informações clínico-epidemiológicas com outros testes para confirmação diagnóstica da doença ${ }^{18}$.

\section{O aumento do}

fígado e do baço (respectivamente,

\section{$94,4 \%$ e $95,5 \%)$}

se seguiram em

maior frequência

depois da febre. A

esplenomegalia é

um dado relevante

para suspeição

clínica da doença,

que costuma

estar ausente nos

imunodeprimidos.
A principal forma de tratamento foi com antimoniato de $\mathrm{N}$-metil glucamina, por ser o medicamento utilizado no país desde a década de 50 como tratamento inicial ${ }^{30}$, semelhante $(64,3 \%)$ ao prescrito aos pacientes com LV de Governador Valadares, Minas Gerais, Brasi ${ }^{31}$.

$\mathrm{O} \mathrm{N}$-metil glucamina é especialmente eficaz no tratamento da leishmaniose visceral (e nas formas cutânea e cutaneomucosa) com regressão das manifestações clínicas e hematológicas da doença. Entretanto, é uma droga que possui cardiotoxicidade e hepatotoxicidade, o que implica em monitoramento do sistema cardiovascular e hepático durante seu uso, especialmente na vigência de co-morbidades, pelo maior risco de óbito por $L V$ nesses pacientes ${ }^{32}$.

$\mathrm{A}$ anfotericina $\mathrm{B}$ lipossomal não foi usada nas crianças com LV internadas no HUJBBB, apesar de relato de menores taxas de letalidade no seu uso sobretudo em menores de um ano de idade, em que a doença apresenta pior prognóstico ${ }^{33,34,35,36,37}$.

A cura ocorreu em 92,7\% da população estudada com taxa de óbito de 7,3\%, respectivamente superiores aos $54,5 \%$ e 1,2\% nos pacientes com LV no Pará ${ }^{14}$. Este maior percentual de cura pode estar relacionado ao HUJBB ser um hospital de referência para doenças infectoparasitárias, com boa qualidade de serviços prestados.

Estudos nacionais, em Bauru, SP e em Campo Grande, MS referiram taxas de letalidade de $8,1 \%$ e de $18,4 \%$, porém, possuem limitação para comparação com o das crianças internadas com LV no HUJBB, pois a amostra incluiu também adultos e com co-morbidades (dentre as quais o HIV/AIDS), que estiveram associadas ao óbito, diferente dos casos atendidos no HUJBB composto por crianças sem comorbidades ${ }^{38,39}$.

O sangramento esteve significativamente associado ao óbito. Segundo a literatura, na dependência do grau de plaquetopenia (em geral, contagem de plaquetas abaixo de $50.000 / \mathrm{mm} 3$ ), o sangramento pode ocorrer sob a forma de epistaxe, equimoses, petéquias, sangramentos digestivos, gengivorragia, sangramentos traumáticos ou associados a procedimentos médico-cirúrgicos ${ }^{33,40,41}$. 
Costa $(2009)^{41}$ observou relação diretamente proporcional entre o número de sítios com sangramento e a letalidade por $\mathrm{LV}$, que ocorre em todas as faixas etárias, mas com maior frequência nas crianças menores de 2 anos. Esse risco de óbito diminuiu para $50 \%$ naquelas entre um e cinco anos. Nas crianças internadas no HUJBBB não foi possível quantificar os sítios de sangramento, impossibilitando assim essa comparação.

As crianças que receberam tratamento inicial com anfotericina B evoluíram significativamente mais para o óbito do que as crianças que iniciaram o tratamento com $\mathrm{N}$-metil glucamina sem que houvesse informações sobre os critérios da escolha terapêutica, outra limitação desse estudo que não permitiu a análise dos critérios epidemiológicos (ida- de), clínico-laboratoriais de gravidade, eventos adversos, dentre outros que norteiam a indicação de anfotericina B como droga de segunda opção para tratamento da $\mathrm{LV}^{42,43}$.

Nessa casuística, a população estudada apresentou média de risco de 1,6, portanto, aquém da pontuação definida pelo SARV para estimativa de risco. Entretanto, comparando-se as crianças que evoluíram para óbito com as que sobreviveram.

Observou-se que as crianças com maior pontuação SARV evoluíram significativamente para óbito comparando-se com àquelas com menor pontuação (3,5 versus 1,4), demonstrando assim a importância de se realizar a avaliação epidemiológica, clínica e laboratorial de cada criança com LV para estabelecer essa pontuação de valor prognóstico ${ }^{41}$.

\section{CONCLUSÕES}

Os casos de LV predominaram na zona rural, a maioria autóctone e infecção primária. As crianças menores de 6 anos e do sexo masculino foram as mais acometidas. A distribuição espacial dos casos de LV foi heterogênea, com maior número de ocorrência no Nordeste Paraense, região endêmica para LV no estado do Pará. O critério laboratorial foi o mais utilizado para confirmação de diagnóstica, principalmente por imunofluorescência indireta. O tratamento com $\mathrm{N}$-metil glucamina foi a terapêutica de eleição mais utilizada, com cura em mais de $90 \%$ dos casos. As crianças com maior pontuação SARV evoluíram significativamente para óbito. A letalidade esteve associada à presença de sangramentos, à plaquetopenia, à falha no tratamento inicial, ao tempo de tratamento e ao escore SARV.

\section{REFERÊNCIAS}

1. DRUGS FOR NEGLECTED DISEASES INITIATIVE - DNDi. 2017. Doenças Negligenciadas: Leishmaniose.

2. Malafaia G, Rodriguês ASL \& Talvani A. (2010). Ética na publicação de pesquisas sobre leishmaniose visceral humana em periódicos nacionais. Rev. Saúde Pública. São Paulo, 2010;45(1):159-165.

3. Prado PF, Rocha MF, Sousa JF, Caldeira DI, Paz GF, Dias EC. Aspectos epidemiológicos da leishmaniose visceral humana e canina em Montes Claros, Estado de Minas Gerais, Brasil, entre 2007 e 2009. Rev. Soc. Bras. Med. Trop.2011;44(5):561-566.

4. BRASIL. Ministério da Saúde. Brasília: Departamento de Vigilância Epidemiológica. Secretaria de Vigilância em Saúde; 2006. Manual de Vigilância da Leishmaniose Visceral. Acesso em 06 jun 2021. Disponível em: https://bvsms.saude.gov.br/bvs/publicacoes/manual_vigilancia_controle_leishmaniose_visceral.pdf

5. Rey LC, Martins CV, Ribeiro HB, Lima AAM. Leishmaniose visceral americana (calazar) em crianças hospitalizadas de área endêmica. J. Pediatr. Rio de Janeiro. 2005;81(1):73-78.

6. Silva RBS, Mendes RS, Santana VL, Souza HC, Ramos CPS, Souza AP, et al. Aspectos epidemiológicos da leishmaniose visceral canina na zona rural do semiárido paraibano e análise de técnicas de diagnóstico. Pesq. Vet. Bras. 2016;36(7):625-629.

7. BRASIL. Ministério da Saúde. Brasília: Secretaria de Vigilância em Saúde. Departamento de Vigilância Epidemiológica; 2014. Manual de Vigilância e Controle da Leishmaniose Visceral. $1^{\text {a }}$ ed. Acesso em 06 jun 2021. Disponível em: http://www.saude. ba.gov.br/wp-content/uploads/2019/05/2014-Manual-de-vigil\%C3\%A2ncia-e-controle-da-leishmaniose-visceral.pdf

8. BRASIL. Ministério da Saúde. Brasília: Secretaria de Vigilância em Saúde. Coordenação Geral de Desenvolvimento da Epidemiologia em Serviços; 2016. Guia Manual de Vigilância em Saúde. $1^{\text {a }}$ ed. 25 f. Acesso em 06 jun 2021. Disponivel em: https://bvsms. saude.gov.br/bvs/publicacoes/guia_vigilancia_saude_3ed.pdf

9. BRASIL. Ministério da Saúde. Brasília: Secretaria de Vigilância em Saúde. Departamento de Vigilância Epidemiológica; 2017. Guia de Vigilância em Saúde. $2^{a}$ ed. Acesso em 06 jun 2021. Disponível em: https://bvsms.saude.gov.br/bvs/publicacoes/guia_ vigilancia_saude_volume_2.pdf

10. Vasconcelos JM, Gomes CG, Sousa A, Teixeira AB, Lima JM. American integumentary leishmaniasis: epidemiological profile, diagnosis and treatment. Braz J of Clin Analyses. 2018;50(3):221-227.

11. BRASIL. Ministério da Saúde. Brasília: Secretaria de Vigilância em Saúde. Coordenação Geral de Desenvolvimento da Epidemiologia em Serviços; 2016. Guia Manual de Vigilância em Saúde. $1^{\text {a }}$ ed., 25 f. Acesso em 06 jun 2021. Disponível em: http://bvsms.saude. gov.br/bvs/publicacoes/guia_vigilancia_saude_1ed_atual.pdf

12. Brandão E, Romero S, Silva MAL, Santos FLN. Neglected tropical diseases in Brazilian children and adolescents: data analysis from 2009 to 2013. Infect Dis of Poverty. 2017;6(154):1-10.

13. Silva BCS, Catete CP, Guimarães LHR, Silva LCT, Guimarães RJPS. Análise espacial da leishmaniose visceral no município de abaetetuba/PA, no período de 2007 a 2015. Cong Abes/Fenasan. 2017;20(17).

14. Silva ES, Gaioso ACI. Visceral leishmaniasis in Pará State. Rev. Para. Med. 2013;27(2):1-8.

15. Müller I, Hailu A, Choi BS, Abebe T, Fuentes JM, Munder M, et al. Age-related alteration of arginase activity impacts on severity 


\section{REFERÊNCIAS}

of leishmaniasis. PLoS Negl Trop Dis. 2008;2(5):235-245.

16. Rodrigues ACM, Melo ACFL, Júnior ADS, Franco SO, Rondon FCM, Bevilaqua, CML. Epidemiologia da leishmaniose visceral no município de Fortaleza, Ceará. Pesq. Vet. Bras. 2017;37(10):1119-1124.

17. Barbosa Ir. Epidemiology of visceral leishmaniasis in the state of Rio Grande do Norte, Brazil. Rev Epidemiol Control Infect. $2013 ; 3(1): 17-21$

18. Teles EJC, Soares DC, Gasparetto D, Veiga N, Fontelles MJP, Garcez LM. Visceral leishmaniasis in Barcarena mining town, Pará State, Brazil: evolution from 2004 to 2008 and bases for epidemiological surveillance. Rev Pan-Amaz Saude. 2014;5(2):21-27.

19. Silveira FT, Lima LVR, Santos TV, Ramos PKS, Campos MB. Reviewingnthe trajectory of American Visceral Leishimaniasis in Brazilian Amazon: from Evandro Chagas to the current days. Rev Pan Amaz Saúde. 2016;(7):15-22.

20. Cordeiro IMCC, Rangel-Vasconcelos LGT, Schwartz G, Oliveira FA. Nordeste Paraense: panorama geral e uso sustentável das florestas secundárias. EDUFRA. 2017;32(49):32-49.

21. Alves E, Souza GS. Marra R. Êxodo e sua contribuição à urbanização de 1950 a 2010. Rev de Política Agrícula. 2011;20(2):80-88.

22. Barbosa MN, Guimarães EADA, Luz ZMPD. Avaliação de estratégia de organização de serviços de saúde para prevenção e controle da leishmaniose visceral. Epidem e Serv de Saúde. 2016;25(3):563-574.

23. Kumar V, Agarwal P, Marwah S, Nigam AS, Tiwari A. Spectrum of clinic hematological profile and its correlation with average parasite density in visceral leishmaniasis. Cytojournal. 2018;15(19).

24. Cunha MA, Antunes AG, Azevedo MME, Maciel RJF, Lacerda HG. Atypical clinical presentation of visceral leishmaniasis: a rare case without splenomegaly. Trop Doc. 2019;49(3):233-234.

25. Van-Griensven J, Diro E. Visceral leishmaniasis. Infect Dis Clin North Am. 2012;26(12):309-322.

26. Antinori S, Cascio A, Parravicini C, Bianchi R, Corbellino M. Leishmaniasis among organ transplant recipients. Lancet Infect Dis, 2008;08(8):191-199.

27. Felipe IMA, Aquino DMC, Kuppinger O, Santos MDC, Rangel MES, Barbosa DS, et al. Infecção por Leishmania em humanos, cães e flebotomíneos em área endêmica de leishmaniose visceral no Maranhão, Brasil. Mem. Inst. Oswaldo Cruz. 2011;106(2).

28. Assis TSM, Braga ASC, Pedras MJ, Barra AMP, Siqueira IC, Costa $\mathrm{CHN}$, et al. Validação do teste imunocromatográfico rápido IT-LEISH ${ }^{\circledR}$ para o diagnóstico da leishmaniose visceral humana. Epidem e Serv de Saúde. 2017;17(2):107-116.

29. Dourado ZF, Delleon SH, Silveira-Lacerda EP, Garcia-Zapata MTA. Panorama histórico do diagnóstico laboratorial da leishmaniose visceral até o surgimento dos testes imunocromatográficos (rK39). Rev de Pat Trop/J of Tropl Pathology. 2017;36(3):205-214.

30. Pastorino AC, Jacob CMA, Oselka GW, Carneiro-Sampaio
MMS. Visceral leishmaniasis: clinical and laboratorial aspects. J Ped. 2002;78(2):120-127.

31. Alves WA, Fonseca DS. Leishmaniose visceral humana: Estudo do perfil clínico-epidemiológico na região leste de Minas Gerais, Brasil. J of Health \& Biol Scienc. 2018;6(2):133-139.

32. Souza MA, Nunes RFF, Viana TC, Marinho MJM, Moreira PVSQ, Pereira WO. Leishmaniose visceral humana: do diagnóstico ao tratamento. Rev. Cien. Saúde Nov. Esp. 2012;10(2):61-69.

33. Gomes LMX, Costa WB, Prado PF, Oliveira-Campos M, Leite MTS. Características clínicas e epidemiológicas da leishmaniose visceral em crianças internadas em um hospital universitário de referência no norte de Minas Gerais, Brasil. Rev Bras Epidemiol. 2009;12(4):549-55.

34. Braga ASC, Junior ACCT, Rabello A. Factors of poor prognosis of visceral leishmaniasis among children under 12 years of age. $A$ retrospective monocentric study in Belo Horizonte, State of Minas Gerais, 2001 a 2005. Rev Soc Bras Med Trop. 2010;46(55):55-59.

35. Pelissari DM, Cechinel MP, Gomes MLS, Lima FES. Treatment of Visceral Leishmaniasis and American Cutaneous Leishmaniasis in Brazil. Epidemiol. Serv. Saúde. 2011;20(1):107-110.

36. Tourinho BD, Figueiredo AF, Lencine FM, Carneiro M. Prognostic factors for death from visceral leishmaniasis in patients treated with liposomal amphotericin B in an endemic state in Brazil. Trans. of The Royal Soc of Trop Med and Hygiene. 2017;111(4):163-171.

37. BRASIL. Ministério da Saúde. Brasília: Secretaria de Vigilância em Saúde. Departamento de Vigilância Epidemiológica; 2017. Manual de vigilância da leishmaniose tegumentar. Brasília - DF, $1^{a}$ ed., 1(191). Acesso em 06 jun 2021. Disponivel em: http:// bvsms.saude.gov.br/bvs/publicacoes/manual_vigilancia_leishmaniose_tegumentar.pdf

38. Ortiz RC, Anversa L. Epidemiologia da leishmaniose visceral em Bauru, São Paulo, no período de 2004 a 2012: um estudo descritivo. Epidemiol. Serv. Saúde. 2015;24(1):97104.

39. Alvarenga DG, Escalda PMF, Da Costa ASV, Monreal MTFD. Leishmaniose visceral: estudo retrospectivo de fatores associados à letalidade. Rev. Soc. Bras. Med. Trop. 2010;43(2):194-7.

40. Queiroz MJ, Alves JG, Correia JB. Leishmaniose visceral: características clínico-epidemiológicas em crianças de área endêmica. J Pediatr. 2004;80(2):141-146.

41. Costa DL. Fatores de prognóstico na leishmaniose visceral: alterações clínicas e laboratoriais associadas à resposta imune, aos distúrbios da coagulação e à morte [tese]. Minas Gerais: Faculdade de Medicina; 2009.

42. Braga ASC. Fatores associados à evolução clínica da leishmaniose visceral em crianças Hospitalizadas em centro de referência de Belo Horizonte, 2001 A 2005. Faculdade Med - UFMG. 2007;07(13):55-59.

43. Leite CEA. Leishamniose Visceral Humana em Pernambuco: Epidemiologia e Gastos com Internações Hospitalares [tese]. Univ Fed de Pernanbuco - UFPE; 2016. 